\title{
REMARKS ON THE EXISTENCE AND THE NON-EXISTENCE OF SOME KIND OF PERIODIC ORBITS FOR HAMILTONIAN SYSTEMS
}

\author{
BY HARUKí YAMADA
}

\section{§ 1. Introduction.}

Let us consider a Hamiltonian system

$$
\dot{\xi}_{k}=H_{\eta_{k}}, \quad \dot{\eta}_{k}=-H_{\xi_{k}}, \quad k=1,2, \cdots, n,
$$

where we assume that the Hamiltonian $H(\xi, \eta)$ is real analytic near the origin $(\xi, \eta)=(0,0)$ and $H_{\xi_{k}}(0,0)=H_{\eta_{k}}(0,0)=0$. Hence $H$ has the following expansion near the origin:

$$
H=\frac{1}{2} t \zeta S \zeta+[\text { higher order terms with respect to } \zeta],
$$

where $\zeta=\left[\begin{array}{l}\xi \\ \eta\end{array}\right] \in \boldsymbol{R}^{2 n}$ and $S$ is a $2 n \times 2 n$ real symmetric matrix. Then the Hamiltonian system (1) can be written in the following form :

$$
\dot{\zeta}=J S \zeta+[\text { higher order terms }] ; \quad J=\left[\begin{array}{rr}
0 & E \\
-E & 0
\end{array}\right],
$$

where $E$ is the $n \times n$ unit matrix.

Recently, various researches have been done on the existence of periodic orbits near the equilibrium point $(0,0)$. It is well known that if, among the eigenvalues $\pm \lambda_{1}, \cdots, \pm \lambda_{n}$ of $J S$, there is a pair of purely imaginary ones

$$
\pm \lambda_{j} \in_{\imath} \boldsymbol{R}, \quad \lambda_{\jmath} \neq 0
$$

such that

$$
\lambda_{k} / \lambda_{j} \notin Z \text { for all } k=1, \cdots, n ; k \neq \jmath,
$$

then, there is a 1-parameter family of periodic orbits which emanate from the equilibrium and whose periods have the values near $2 \pi /\left|\lambda_{j}\right|$. This is a theorem of Liapunov. (For precise statement and proof of this theorem see e.g. [8] $\S 16$ ). Though it is known that the condition (4) is essential for the existence of such

Received March 24, 1980 
family of periodic orbits, it is often possible to remove the condition (5) or replace it by some other conditions. (see e. g. [1], [2], [3], [5], [9] and references cited there). On the contrary, it seems that [7] is the only known general result for the non-existence of (some kind of) periodic orbits. In [7] it is proved, for example, that if there is only one pair of purely imaginary eigenvalues $\pm \lambda_{1} \in i \boldsymbol{R}$, $\lambda_{1} \neq 0$, and $\operatorname{Re} \lambda_{j} \neq 0$ for all $j=2, \cdots, n$, then in a sufficiently small neighbourhood of the origin, there are no periodic orbits other than those whose existence is guaranteed by the theorem of Liapunov.

The aim of this note is to present a somewhat systematic method of construction of examples for the non-existence of (some kind of) periodic orbits when all the eigenvalues of JS are purely imaginary. Before doing so, in $\S 2$, we review on the normal forms and reality conditions for Hamiltonian systems. In $\S 3$, we use our method to construct some examples in the case of two degrees of freedom. We also prove a simple existence theorem of infinitely many periodic orbits with arbitrary long periods when the Hamiltonian can be transformed into a normal form. In $\S 4$, we indicate a generalization to the case of several degrees of freedom.

I wish to thank to Mr. H. Ito for valuable discussions. This work was supported by Grants-in-Aid for Scientific Research from the Ministry of Education, Science and Culture (No. 454025).

\section{$\S 2$. Normal forms and reality conditions. (cf. [8], §§. 15, 30).}

In what follows, for simplicity, we shall assume that all the eigenvalues of JS are non-zero purely imaginary numbers. Then we can take a linear canonical transformation

$$
\zeta=C w ; \quad w=\left(\begin{array}{l}
u \\
v
\end{array}\right) \in \boldsymbol{R}^{2 n},
$$

to transform the Hamiltonian (2) into the following form:

$$
H=H(w)=H(u, v)=\sum_{j=1}^{n} \lambda_{j} u_{j} v_{j}+[\text { higher order terms }],
$$

where the reality condition is

$$
v_{j}=i \bar{u}_{\jmath}, \quad \jmath=1, \cdots, n .
$$

In other words, if we find a solution $w(t)=\left(\begin{array}{c}u(t) \\ v(t)\end{array}\right)$ of

$$
\dot{u}_{k}=H_{v_{k}}, \quad \dot{v}_{k}=-H_{u_{k}}, \quad k=1, \cdots, n
$$

such that $(7)$ is true for all $t$, then the function $\zeta(t)$ defined by

$$
\zeta(t)=C w(t)
$$


is a real valued solution of (1). Further, if we take

$$
u_{k}=\frac{1}{\sqrt{2}}\left(p_{k}+\imath q_{k}\right), \quad v_{k}=\frac{1}{\sqrt{2}}\left(q_{k}+\imath p_{k}\right)
$$

the transformation $\left(\begin{array}{l}u \\ v\end{array}\right) \rightarrow\left(\begin{array}{l}p \\ q\end{array}\right)$ is a canonical transformation and the reality condition (7) becomes

$$
p_{k} \text { and } q_{k} \text { are real valued for all } k=1, \cdots, n \text {. }
$$

Then we have $u_{k} v_{k}=i\left(p_{k}^{2}+q_{k}^{2}\right) / 2$ and

$$
H=\sum_{k=1}^{n} i \lambda_{k}\left(p_{k}^{2}+q_{k}^{2}\right) / 2+\cdots .
$$

If we take a further transformation

$$
x_{k}=\frac{1}{\sqrt{2}}\left(p_{k}-\imath q_{k}\right), \quad y_{k}=\frac{1}{\sqrt{2}}\left(p_{k}+i q_{k}\right)=\bar{x}_{k},
$$

(which is not a canonical transformation), the reality condition becomes

$$
x_{k}=\bar{y}_{k}, \quad k=1, \cdots, n,
$$

and the Hamiltonian system (8) becomes

$$
x_{k}=i H_{y_{k}}, \quad \dot{y}_{k}=-i H_{x_{k}}, \quad k=1, \cdots, n,
$$

where

$$
H=\sum_{k=1}^{n} i \lambda_{k} x_{k} y_{k}+\cdots
$$

Note that under the reality condition (11), we have

$$
x_{k} y_{k}=x_{k} \bar{x}_{k}=\left|x_{k}\right|^{2} \geqq 0 \text {. }
$$

Further note that, though $\pm \lambda_{k}$ are eigenvalues of $J S$, the signs of $i \lambda_{k}$ in (13) are determined uniquely if we impose the reality condition (11). In the following we shall consider the Hamiltonian system in the form (12), (13) under the reality condition (11). Further, in what follows, for the simplicity, we shall say that a function $f=f(x, y)$ of $x_{k}$ and $y_{k}$ is real valued if it is real valued when we replace $y_{k}$ by $\bar{x}_{k}$ (i.e. if the reality condition (11) is valid). Of course the Hamiltonian (13) is a real valued function.

Our method of construction of examples relies on a fact that if there is a real valued function $F(x, y)$ such that, for any solution $\left(\begin{array}{l}x(t) \\ y(t)\end{array}\right)$ of (12),

$$
\frac{d}{d t} F(x, y)=\varphi(x, y) \geqq 0 \text { for all }(x, y) \text { with }(11) \text {, }
$$


then there are no periodic solutions other than those with $\varphi(x(t), y(t)) \equiv 0$.

\section{$\S 3$. The case of two degrees of freedom.}

In this section we restrict ourselves to the case of $n=2$ in (1):

$$
\dot{\xi}_{k}=H_{\eta_{k}}, \quad \dot{\eta}_{k}=-H_{\xi_{k}}, \quad k=1,2 .
$$

We assume that the eigenvalues $\pm \lambda_{1}, \pm \lambda_{2}$ of $J S$ are non-zero purely imaginary numbers. It is known that $([8], \S 30)$, if further

$$
\lambda_{1} g_{1}+\lambda_{2} g_{2}=0, \quad g_{1}, g_{2} \in \boldsymbol{Z} \Leftrightarrow g_{1}=g_{2}=0,
$$

is valid, we can transform $H$ by the composition of a formal canonical transformation (which is defined by a generating function expressed by a formal power series) and transformations (9) and (10) to the following form:

$$
H=i\left(\lambda_{1} x_{1} y_{1}+\lambda_{2} x_{2} y_{2}\right)+\frac{1}{2} R x_{1}^{2} y_{1}^{2}+S x_{1} y_{1} x_{2} y_{2}+\frac{1}{2} T x_{2}^{2} y_{2}^{2}+h\left(\omega_{1}, \omega_{2}\right),
$$

where $R, S$ and $T$ are real constants and $h$ is a real valued formal power series in products $\omega_{1}=x_{1} y_{1}, \omega_{2}=x_{2} y_{2}$ alone, the reality condition is the same as (11). Unfortunately, it is known that, in general, one has divergence (see [6]). But if it happens that it is convergent, we have the following result.

THEOREM 1. Suppose that the Hamiltonian can be transformed into the form (16) by a convergent transformation. (We do not necessarily impose the condition (15)). Suppose further that

$$
R \lambda_{2} \neq S \lambda_{1} \text { or } S \lambda_{2} \neq T \lambda_{1}
$$

is valid. Then, for any small $\varepsilon>0$ and large $N>0$, there are infintely many periodic orbits with primitive period larger than $N$ in the e-neighbourhood of the origin $0 \in \boldsymbol{R}^{4}$.

Proof. If we write $\omega_{1}=x_{1} y_{1}, \omega_{2}=x_{2} y_{2}$, we have, by (12), the Hamiltonian system in the form

$$
\dot{x}_{k}=i H_{\omega_{k}} x_{k}, \quad \dot{y}_{k}=-i H_{\omega_{k}} y_{k},
$$

from which it follows that $\dot{\omega}_{k} \equiv 0, k=1,2$. Hence the $\omega_{k}$ are (real valued) integrals of the system and hence $H_{\omega_{k}}(x, y)$ are independent of $t$ if we take any solution $x_{k}=x_{k}(t), y_{k}=y_{k}(t)$ of (18). Thus we can integrate (18) in the form

$$
x_{k}=c_{k} e^{i \omega_{\omega_{k}} t}, \quad y_{k}=d_{k} e^{-i H_{\omega_{k}} t} .
$$

If we take $d_{k}=\bar{c}_{k}$ and use the fact that $H$, and hence $H_{\omega_{k}}$, are real valued, we know that the solution (19) satisfies the reality conditions for all $t$. Note that, 
in this case, $x_{k} y_{k}=\omega_{k}=c_{k} d_{k}=\left|c_{k}\right|^{2}$ (constant). The general solution of (14) can be written by a linear combination of $x_{k}(t), y_{k}(t), k=1,2$, and $x_{k}(t), y_{k}(t)$ are periodic functions with primitive period $2 \pi /\left|H_{\omega_{k}}\right|$. Thus if we are able to take $\left(c_{1}, c_{2}\right) \in C^{2}$ in the $\varepsilon$-neighbourhood of the origin $(0,0) \in C^{2}$ such that

$$
2 \pi /\left|H_{\omega_{1}}\right|: 2 \pi /\left|H_{\omega_{2}}\right|=p: q,
$$

where $p$ and $q$ are relatively prime positive integers, we have a periodic orbit with primitive period

$$
2 q \pi /\left|H_{\omega_{1}}\right|=2 p \pi /\left|H_{\omega_{2}}\right| .
$$

Now let us consider the ratio

$$
\frac{H_{\omega_{1}}}{H_{\omega_{2}}}=\frac{\rho_{1}+R \omega_{1}+S \omega_{2}+\cdots}{\rho_{2}+S \omega_{1}+T \omega_{2}+\cdots}
$$

where $\rho_{1}=i \lambda_{1}, \rho_{2}=i \lambda_{2}$ are non-zero real numbers and the dots $\cdots$ indicate the terms of order $\geqq 2$ with respect to $\omega_{1}, \omega_{2}$. If we take $\omega_{1}=k s, \omega_{2}=l s$ with $k, l>0$, $k^{2}+l^{2}=1, s>0$, and put

we have

$$
f(s)=\frac{H_{\omega_{1}}(k s, l s)}{H_{\omega_{2}}(k s, l s)}=\frac{\rho_{1}+(k R+l S) s+0\left(s^{2}\right)}{\rho_{2}+(k S+l T) s+0\left(s^{2}\right)},
$$

$$
f^{\prime}(0)=\frac{1}{\rho_{2}^{2}}\left[\left(R \rho_{2}-S \rho_{1}\right) k+\left(S \rho_{2}-T \rho_{1}\right) l\right] .
$$

Thus if (17) is valid, we have for almost all values $k, l, f^{\prime}(0) \neq 0$ and hence $f(s)$ is a monotone function near $s=0$. Thus there is a constant $\delta_{0}>0$ such that for any $\delta$ with $0<\delta<\delta_{0},\{f(s) ; 0 \leqq s \leqq \delta\}$ is a closed interval with inner points in $\boldsymbol{R}$ and it contains $\rho_{1} / \rho_{2}$ as a terminal point. It is easy to see that in this interval there are infinitely many rational numbers $q / p$ with $(p, q)=1$ and $|p|,|q|>M$ where $M$ is an arbitrary fixed (large) number. If we take $s$ such that $f(s)=q / p$ and take $\omega_{1}, \omega_{2}$ as $\omega_{1}=k s, \omega_{2}=l s$, we have a desired long periodic orbit

with period

$$
\begin{array}{ll}
x_{1}=\sqrt{\omega_{1}} e^{i H_{\omega_{1}} t}, & x_{2}=\sqrt{\omega_{2}} e^{i H \omega_{2} t}, \\
y_{1}=\sqrt{\omega_{1}} e^{-i H_{\omega_{1}} t}, & y_{2}=\sqrt{\omega_{2}} e^{-i H_{\omega_{2}} t},
\end{array}
$$

$$
\frac{2 q \pi}{H_{\omega_{1}}}=\frac{2 p \pi}{H_{\omega_{2}}}\left(\sim \frac{2 q \pi}{\rho_{1}}, \frac{2 p \pi}{\rho_{2}}\right) .
$$

This proves the theorem.

Remark 1. It is clear that if

$$
R \lambda_{2}^{2}-2 S \lambda_{1} \lambda_{2}+T \lambda_{1}^{2} \neq 0,
$$

that is, if the polynomial $R \xi^{2}+2 S \xi \eta+T \eta^{2}$ can not be divisible by $\lambda_{1} \xi+\lambda_{2} \eta$, the 
condition (17) is valid.

Now if $\lambda_{1} / \lambda_{2}, \lambda_{2} / \lambda_{1} \notin Z$, it always exist two 1 -parameter family of periodic orbits with periods $\sim 2 \pi /\left|\lambda_{1}\right|$ and $\sim 2 \pi /\left|\lambda_{2}\right|$ which are guaranteed by the theorem of Liapunov. The above theorem says that if further $H$ can be transformed into the normal form (16) and the condition (17) is valid, there are infinitely many long periodic orbits other than the above ones. On the other hand, for

$$
H=i\left(\lambda_{1} x_{1} y_{1}+\lambda_{2} x_{2} y_{2}\right), \quad \lambda_{1} / \lambda_{2} \notin \boldsymbol{Q},
$$

there are no periodic orbits at all other than those cited above. It is likely that under the conditions (15) and (17) (we do not impose the condition that the transformation into the normal form (16) is convergent), there always be infinitely many long periodic orbits near the origin. But we don't know whether this is true or not.

If $\lambda_{1} / \lambda_{2} \in \boldsymbol{Q}$, on the other hand, all the solutions for (20) are periodic. But in general these long periodic orbits may disappear by some perturbations for $H$. To see this we start from somewhat general context.

Let us consider the Hamiltonian

$$
H=i\left(\lambda_{1} x_{1} y_{1}+\lambda_{2} x_{2} y_{2}\right)+\frac{1}{2} R x_{1}^{2} y_{1}^{2}+S x_{1} y_{1} x_{2} y_{2}+\frac{1}{2} T x_{2}^{2} y_{2}^{2}+h(x, y),
$$

where $h(x, y)$, which represents higher order terms, is not necessarily a function in $\omega_{1}$ and $\omega_{2}$ only. Then the Hamiltonian system is

$$
\begin{aligned}
& \dot{x}_{1}=i H_{y_{1}}=i\left(\rho_{1}+R x_{1} y_{1}+S x_{2} y_{2}\right) x_{1}+i h_{y_{1}}, \\
& \dot{x}_{2}=i H_{y_{2}}=i\left(\rho_{2}+S x_{1} y_{1}+T x_{2} y_{2}\right) x_{2}+i h_{y_{2}},
\end{aligned}
$$

where $\rho_{1}=i \lambda_{1}, \rho_{2}=i \lambda_{2}$. Note that other two equations can be given by taking the complex conjugates in (22) and replacing $\bar{x}_{k}$ and $\bar{y}_{k}$ by $y_{k}$ and $x_{k}$ respectively. Now take real valued functions $f(x, y)$ and $g(x, y)$ and put

$$
s=s(x, y)=\frac{g(x, y)}{f(x, y)} .
$$

Then, if we take $x_{k}, y_{k}$ as a solution of (22), we have

$$
\dot{s}=\frac{1}{f^{2}}(\dot{g} f-\dot{f} g)
$$

and

$$
\dot{g} f-\dot{f} g=\left(g_{x_{1}} f-f_{x_{1}} g\right) \dot{x}_{1}+\left(g_{x_{2}} f-f_{x_{2}} g\right) \dot{x}_{2}+\left(g_{y_{1}} f-f_{y_{1}} g\right) \dot{y}_{1}+\left(g_{y_{2}} f-f_{y_{2}} g\right) \dot{y}_{2} .
$$

If we put

$$
\begin{array}{ll}
A=g_{x_{1}} f-f_{x_{1}} g, & B=g_{y_{1}} f-f_{y_{1}} g, \\
C=g_{x_{2}} f-f_{x_{2}} g, & D=g_{y_{2}} f-f_{y_{2}} g,
\end{array}
$$


we have

$$
\begin{aligned}
\dot{g} f-\dot{f} g= & i\left(x_{1} A-y_{1} B\right)\left(\rho_{1}+R x_{1} y_{1}+S x_{2} y_{2}\right)+i\left(x_{2} C-y_{2} D\right)\left(\rho_{2}+S x_{1} y_{1}+T x_{2} y_{2}\right) \\
& +i\left(h_{y_{1}} A-h_{x_{1}} B+h_{y_{2}} C-h_{x_{2}} D\right) .
\end{aligned}
$$

Note that the condition $\lambda_{1} / \lambda_{2}=\rho_{1} / \rho_{2} \in \boldsymbol{Q}$ reflects the fact that we can take appropriate $f$ and $g$ to cancel out the term

$$
i \rho_{1}\left(x_{1} A-y_{1} B\right)+i \rho_{2}\left(x_{2} C-y_{2} D\right) .
$$

Using the above formulations we shall prove the following fact.

THEOREM 2. Suppose that $\lambda_{1} / \lambda_{2} \in \boldsymbol{Q}, \lambda_{2} / \lambda_{1}, \lambda_{1} / \lambda_{2} \notin Z$ and $\lambda_{1} / \lambda_{2}<0$. Then there is a Hamiltonian $H$ with quadratic term $i \lambda_{1} x_{1} y_{1}+i \lambda_{2} x_{2} y_{2}$ and $R, S, T \neq 0$ such that there are no periodic orbits other than those two 1-parameter families whose existence is guaranteed by the theorem of Liapunov.

Proof. By scaling the time variable $t$ appropriately, it is enough to prove the theorem when

$$
\left.\lambda_{1}=-\imath p, \quad \lambda_{2}=\imath q \quad \text { (i. e. } \rho_{1}=p, \rho_{2}=-q\right),
$$

where $p$ and $q$ are positive integers and $(p, q)=1, p \neq 1, q \neq 1$. Now consider the Hamiltonian in the form (21) with

$$
h=x_{1}^{q} x_{2}^{p}+y_{1}^{q} y_{2}^{p}
$$

and take $f=1, g=\frac{1}{2}\left(x_{1}^{q} x_{2}^{p}-y_{1}^{q} y_{2}^{p}\right)$ in (23). Then we have

$$
\begin{aligned}
\dot{s}=\dot{g}= & q\left(x_{1}^{q} x_{2}^{p}+y_{1}^{q} y_{2}^{p}\right)\left(p+R x_{1} y_{1}+S x_{2} y_{2}\right) \\
& +p\left(x_{1}^{q} x_{2}^{p}+y_{1}^{q} y_{2}^{p}\right)\left(-q+S x_{1} y_{1}+T x_{2} y_{2}\right) \\
& +2\left(x_{1} y_{1}\right)^{q-1}\left(x_{2} y_{2}\right)^{p-1}\left(p^{2} x_{1} y_{1}+q^{2} x_{2} y_{2}\right) \\
= & \left\{(q R+p S) x_{1} y_{1}+(q S+p T) x_{2} y_{2}\right\}\left(x_{1}^{q} x_{2}^{p}+y_{1}^{q} y_{2}^{p}\right) \\
& +2\left(x_{1} y_{1}\right)^{q-1}\left(x_{2} y_{2}\right)^{p-1}\left(p^{2} x_{1} y_{1}+q^{2} x_{2} y_{2}\right) .
\end{aligned}
$$

Thus if we take $R, S$ and $T$ such that

$$
q R+p S=0, \quad q S+p T=0,
$$

we have

$$
\dot{s}=2\left(x_{1} y_{1}\right)^{q-1}\left(x_{2} y_{2}\right)^{p-1}\left(p^{2} x_{1} y_{1}+q^{2} x_{2} y_{2}\right) \geqq 0,
$$

where the equality is valid identically in time $t$ if and only if

$$
x_{1} \equiv y_{1} \equiv 0 \quad \text { or } \quad x_{2} \equiv y_{2} \equiv 0 \text {. }
$$


But if $x_{2} \equiv y_{2} \equiv 0$ we have the Hamiltonian system

$$
\begin{array}{ll}
\dot{x}_{1}=i\left(p+R x_{1} y_{1}\right) x_{1}, & x_{2}=0, \\
\dot{y}_{1}=-i\left(p+R x_{1} y_{1}\right) y_{1}, & \dot{y}_{2}=0,
\end{array}
$$

which has the 1-parameter family of periodic orbits

$$
\begin{array}{ll}
x_{1}=\rho e^{\imath\left(p+R \rho^{2}\right) t}, & x_{2} \equiv 0, \\
y_{1}=\rho e^{-\imath\left(p+R \rho^{2}\right) t}, & y_{2} \equiv 0,
\end{array}
$$

where $\rho$ is a non-negative real parameter. Similarly, if $x_{1} \equiv y_{1} \equiv 0$, we have another 1-parameter family of periodic orbits

$$
\begin{array}{ll}
x_{1} \equiv 0, & x_{2}=\rho e^{\imath\left(-q+S \rho^{2}\right) t}, \\
y_{1} \equiv 0, & y_{2}=\rho e^{-\imath\left(-q+S \rho^{2}\right) t} .
\end{array}
$$

These orbits have periods $2 \pi /\left(p+R \rho^{2}\right)$ and $2 \pi /\left(q-S \rho^{2}\right)$ respectively. (These orbits are those which are given by the theorem of Liapunov). By (25) all other orbits cannot be periodic. This proves the theorem.

Remark 2. The condition (24) can be written as

$$
\lambda_{2} R=\lambda_{1} S, \quad \lambda_{2} S=\lambda_{1} T .
$$

If one of these equality breaks down and if $h(x, y)$ is replaced by a function in $\omega_{1}$ and $\omega_{2}$, we have, by Theorem 1 , there are infinitely many periodic orbits with arbitrary large primitive periods.

Remark 3. For the Hamiltonian which was given in the proof of Theorem 2, the result in Theorem 2 is valid globally in $\boldsymbol{R}^{4}$.

Remark 4. We do not know whether, if $\lambda_{1} / \lambda_{2}>0$, we can construct a similar example as in Theorem 2 or not. But if we consider non-Hamiltonian system, we are able to do so. In fact, e.g., consider the system

$$
\begin{aligned}
& \dot{x}_{1}=i\left(p+R x_{1} y_{1}+S x_{2} y_{2}\right) x_{1}-x_{1}^{q+1} y_{2}^{p}, \\
& \dot{x}_{2}=i\left(q+S x_{1} y_{1}+T x_{2} y_{2}\right) x_{2}+x_{1}^{q} y_{2}^{p-1}, \\
& \dot{y}_{1}=-i\left(p+R x_{1} y_{1}+S x_{2} y_{2}\right) y_{1}-y_{1}^{q+1} x_{2}^{p}, \\
& \dot{y}_{2}=-i\left(q+S x_{1} y_{1}+T x_{2} y_{2}\right) y_{2}+y_{1}^{q} x_{2}^{p-1} .
\end{aligned}
$$

Then if we take

$$
s=\frac{g}{f}=\frac{y_{1}^{q} x_{2}^{p}+x_{1}^{q} y_{2}^{p}}{x_{1}^{q} y_{1}^{q}}
$$

we have 


$$
\begin{aligned}
\dot{s}= & \imath q\left(x_{1} y_{1}\right)^{-q}\left(x_{1}^{q} y_{2}^{p}-y_{1}^{q} x_{2}^{p}\right)\left(p+R x_{1} y_{1}+S x_{2} y_{2}\right) \\
& -\imath p\left(x_{1} y_{1}\right)^{-q}\left(x_{1}^{q} y_{2}^{p}-y_{1}^{q} x_{2}^{p}\right)\left(q+S x_{1} y_{1}+T x_{2} y_{2}\right)+2\left(x_{2} y_{2}\right)^{p-1}\left(q x_{2} y_{2}+p\right) .
\end{aligned}
$$

Accordingly, if we take $R, S$, and $T$ as

$$
q R-p S=0, q S-p T=0,
$$

we have

$$
\dot{s}=2\left(x_{2} y_{2}\right)^{p-1}\left(q x_{2} y_{2}+p\right) \geqq 0
$$

and the equality is valid identically only if $x_{2} \equiv y_{2} \equiv 0$. Note that the above calculation is valid only if $x_{1} \neq 0, y_{1} \neq 0$. Thus this system has no periodic orbits other than two 1-parameter families of periodic orbits which correspond with $x_{1} \equiv y_{1} \equiv 0$ or $x_{2} \equiv y_{2} \equiv 0$. Note that the third and the fourth equations are consequences of the first and the second equations and the reality condition. Thus the system (26) has a meaning as the system in $\boldsymbol{R}^{4}$. Unfortunately (26) is not a Hamiltonian system.

Remark 5. In [2], it is shown that if we assume some conditions for the coefficients of higher order terms of the Hamiltonian, we have the existence of some kind of long periodic orbits other than those whose existence is guaranteed by the theorem of Liapunov. Of course our examples do not satisfy this condition.

In the case when $\lambda_{1} / \lambda_{2} \in Z$ we can prove easily the following theorem.

THEOREM 3. Suppose that $\lambda_{1} / \lambda_{2} \in Z$ and $\lambda_{1} / \lambda_{2}<0$. Then there is a Hamiltonian with $R, S, T \neq 0$, for which there are no periodic orbits other than one 1-parameter family whose existence is guaranteed by the theorem of Liapunov.

Proof. If $\lambda_{1} / \lambda_{2} \neq-1$, we have only to take $q=1$ in the proof of Theorem 2 . Then if we take $R, S$, and $T$ as

$$
R+p S=0, \quad S+p T=0,
$$

we have

$$
\dot{s}=2\left(x_{2} y_{2}\right)^{p-1}\left(p^{2} x_{1} y_{1}+x_{2} y_{2}\right) \geqq 0
$$

and there must only be one 1-parameter family of periodic orbits corresponding $x_{2} \equiv y_{2} \equiv 0$. Note that the Hamiltonian can be written as

$$
H=p x_{1} y_{1}-x_{2} y_{2}+\frac{1}{2} R x_{1}^{2} y_{1}^{2}+S x_{1} y_{1} x_{2} y_{2}+\frac{1}{2} T x_{2}^{2} y_{2}^{2}+x_{1} x_{2}^{p}+y_{1} y_{2}^{p} .
$$

Thus unless $p=1$, the degree of $x_{1} x_{2}^{p}+y_{1} y_{2}^{p}$ is $p+1 \geqq 3$. If $\lambda_{1} / \lambda_{2}=-1$, we may take

$$
H=x_{1} y_{1}-x_{2} y_{2}+\left(x_{1} y_{1}+x_{2} y_{2}\right)\left(x_{1} x_{2}+y_{1} y_{2}\right)
$$


and $s=\left(x_{1} x_{2}-y_{1} y_{2}\right) / i$ (This is due to [4]). This proves the theorem.

Remark 6. In [5], it is proved that for the Hamiltonian (21), if $S+p T \neq 0$, $p \geqq 4$, there is a 1-parameter family of periodic orbits with primitive periods $\sim 2 \pi$ near the origin. (Note that by the theorem of Liapunov it always exists a 1-parameter family of periodic orbits with primitive periods $\sim 2 \pi / p$ ). Our example in the proof of Theorem 3 was constructed under the condition (28). We do not know whether we can construct an example of non-existence of long periodic orbits (with periods $\sim 2 \pi$ ) assuming only that $S+p T=0$ on the fourth order terms of the Hamiltonian.

Remark 7. In [9], it is proved that for the Hamiltonian $H=x_{1} y_{1}+p x_{2} y_{2}$ $+h(x, y)$, where $h$ contains only terms of order $\geqq 3$ and $p$ is any positive real number, there are at least two periodic orbits on each energy surfaces $H=\varepsilon$ where $\varepsilon>0$ are sufficiently small real numbers. Thus the assumption $\lambda_{1} / \lambda_{2}<0$ in Theorem 3 is not so restrictive.

Remark 8. By investigation on the properties of $\dot{s}$ in the proof of Theorem 3 , we have easily that, if $S+p T=0$, there are no periodic orbits for (29) in a domain near the plane $x_{2}=y_{2}=0$ in which there exist long periodic orbits when $S+p T \neq 0, p \geqq 4$.

\section{§4. A generalization.}

A part of our method in $\S 3$ can be extended to the case of several degrees of freedom. We shall mention only one example which is connected with a result of [9] (This is also a straight forward generalization of an example in $[4])$.

Consider the Hamiltonian of $2 n$-degrees of freedom :

$$
H=\sum_{k=1}^{n} \rho_{k} x_{k} y_{k}-\sum_{k=1}^{n} \rho_{k} x_{n+k} y_{n+k}+\left(\sum_{l=1}^{2 n} x_{l} y_{l}\right) \sum_{k=1}^{n}\left(x_{k} x_{n+k}+y_{k} y_{n+k}\right) \text {, }
$$

where $\rho_{k}$ are non-zero real numbers. Then there are no periodic orbits at all for (30) other than the equilibrium. Let us prove this in the following manner:

The Hamiltonian system for (30) is

$$
\begin{array}{r}
x_{\jmath}=i H_{y_{\jmath}}=i\left[\rho_{\jmath} x_{j}+x, \sum_{k=1}^{n}\left(x_{k} x_{n+k}+y_{k} y_{n+k}\right)+y_{n+j}\left(\sum_{l=1}^{2 n} x_{l} y_{l}\right)\right], \\
\dot{x}_{n+\jmath}=i H_{y_{n+\jmath}}=i\left[-\rho_{\jmath} x_{n+j}+x_{n+\jmath} \sum_{k=1}^{n}\left(x_{k} x_{n+k}+y_{k} y_{n+k}\right)+y_{\jmath}\left(\sum_{l=1}^{2 n} x_{l} y_{l}\right)\right], \\
\jmath=1, \cdots, n .
\end{array}
$$


Other $2 n$ equations for $y_{\jmath}, y_{n+\jmath}, \jmath=1, \cdots, n$, can be given by conjugating (31) and replacing $\bar{x}_{l}$ and $\bar{y}_{l}, l=1, \cdots, 2 n$, by $y_{l}$ and $x_{l}$ respectively. If we take a real valued function

$$
s=\frac{1}{\imath} \sum_{j=1}^{n}\left(x, x_{n+j}-y_{\jmath} y_{n+j}\right),
$$

we have

$$
\dot{s}=2\left\{\sum_{j=1}^{n}\left(x_{\jmath} x_{n+j}+y_{\jmath} y_{n+j}\right)\right\}^{2}=2\left(\sum_{l=1}^{2 n} x_{l} y_{l}\right)^{2} \geqq 0 \text {. }
$$

The equality is valid identically only if $x_{l} \equiv y_{l} \equiv 0$ for all $l=1, \cdots, 2 n$. This proves that the only periodic orbit for (31) is an equilibrium.

Remark 1. By a similar method, it is possible to make an example of Hamiltonian with the form

$$
H=\sum_{k=1}^{n} \rho_{k} x_{k} y_{k}-\sum_{k=1}^{n+m} \rho_{k} x_{n+k} y_{n+k}+\cdots
$$

with $\rho_{k} \in \boldsymbol{R}, \rho_{k} \neq 0, k=1, \cdots, n+m, n>0, m \geqq 0 ; \rho_{k} / \rho_{n+l} \notin \boldsymbol{Z}$ for all $k=1, \cdots, n$, $l=1, \cdots, m ; \rho_{n+l} / \rho_{n+s} \notin \boldsymbol{Z}$ for all $l, s=1, \cdots, m, l \neq s$, for which the Hamiltonian system has only $m$ 1-parameter families of periodic orbits.

Remark 2. In [1], the following result is proved: Let

$$
H=\sum_{k=1}^{2 n+m} \rho_{k} x_{k} y_{k}+h(x, y) \text {, }
$$

where $\rho_{k}>0$ for $k=1, \cdots, n, \rho_{k}<0$ for $k=n+1, \cdots, 2 n+m$, and where $h$ represents the terms of order $\geqq 3$. Suppose that there is a number $T \in \boldsymbol{R}$ such that

$$
T /\left(2 \pi / \rho_{k}\right) \in \boldsymbol{Z} \text { for all } k=1, \cdots, 2 n+m \text {. }
$$

Then there is a left neighbourhood $I_{l}$ and a right neighbourhood $I_{r}$ of $T \in \boldsymbol{R}$ and a pair of integers $a, b \geqq 0$ with $a+b=m$ such that, for all $\tau \in I_{l}$ (resp. $I_{r}$ ), the Hamiltonian system for (32) possesses at least $a$ (resp. $b$ ) distinct non-trivial $\tau$-periodic orbits.

Our example shows that the condition $m>0$ is essential for the existence of periodic orbits when we do not impose any additional conditions on the coefficients in $h(x, y)$ (i. e. terms of order $\geqq 3$ ).

\section{REFERENCES}

[1] E. R. Fadell and P.H. Rabinowitz, Generalized cohomological index theories for Lie group actions with an application to bifurcation questions for Hamiltonian systems, Inv. Math., 45 (1978), 139-174.

[2] J. Henrard, Periodic orbits emanating from a resonant equilibrium, Celes. Mech., 
1 (1970), 437-466.

[3] J.K. Moser, Periodic orbits near an equilibrium and a theorem by Alan Weinstein, Comm. Pure Appl. Math., 29 (1976), 727-747.

[4] J. K. Moser, Addendum to [3], Comm. Pure Appl. Math., 31 (1978), 529-530.

[5] D.S. SchmidT, Hopf bifurcation theorem and the center theorem of Liapunov with resonance case, J. Math. Anal. Appl., 63 (1978), 354-370.

[6] C.L. Siegel, Über die Existenz einer Normalform analytischer Hamiltonscher Differentialgleichungen in der Nähe einer Gleichgewıchtslösung, Math. Ann., 128 (1954), 144-170.

[7] C. L. Siegel, Periodische Lösungen von Differentıalgleıchungen, Nachr. Akad. Wiss. Göttingen, Math-Phys. Kl. II, (1971), 261-283.

[8] C.L. Siegel ANd J. K. Moser. Lectures on Celestial Mechanics, Springer, 1971.

[9] A. Weinstein, Normal modes for non-linear Hamiltonian systems, Inv. Math. 20 (1973), 47-57.

Department of MATHEMATics

TOKYo INSTITUTE OF TECHNOLOGY 